TITLE:

\title{
Formation of a hybrid plasmonic waveguide mode probed by dispersion measurement
}

\section{$\operatorname{AUTHOR}(\mathrm{S}):$}

Saito, H.; Kurata, H.

\section{CITATION:}

Saito, H....[et al]. Formation of a hybrid plasmonic waveguide mode probed by dispersion measurement. Journal of Applied Physics 2015, 117(13): 133107.

\section{ISSUE DATE:}

2015-04-07

URL:

http://hdl.handle.net/2433/198836

\section{RIGHT:}

(c) 2015 American Institute of Physics. This article may be downloaded for personal use only. Any other use requires prior permission of the author and the American Institute of Physics. 


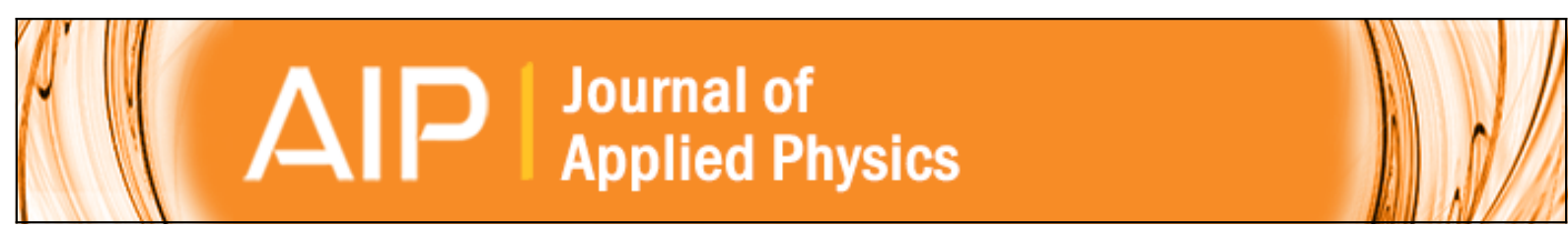

\section{Formation of a hybrid plasmonic waveguide mode probed by dispersion measurement}

H. Saito and H. Kurata

Citation: Journal of Applied Physics 117, 133107 (2015); doi: 10.1063/1.4916800

View online: http://dx.doi.org/10.1063/1.4916800

View Table of Contents: http://scitation.aip.org/content/aip/journal/jap/117/13?ver=pdfcov

Published by the AIP Publishing

\section{Articles you may be interested in}

Numerical investigation of optical Tamm states in two-dimensional hybrid plasmonic-photonic crystal nanobeams J. Appl. Phys. 116, 043106 (2014); 10.1063/1.4891222

Demonstration of low-loss on-chip integrated plasmonic waveguide based on simple fabrication steps on siliconon-insulator platform

Appl. Phys. Lett. 101, 041117 (2012); 10.1063/1.4739523

Thermo-optic plasmo-photonic mode interference switches based on dielectric loaded waveguides Appl. Phys. Lett. 99, 241110 (2011); 10.1063/1.3670500

Optical performance of single-mode hybrid dielectric-loaded plasmonic waveguide-based components Appl. Phys. Lett. 96, 221103 (2010); 10.1063/1.3437088

Long-range surface plasmon-polariton waveguides and devices in lithium niobate J. Appl. Phys. 101, 113114 (2007); 10.1063/1.2739300

Frustrated by

old technology?

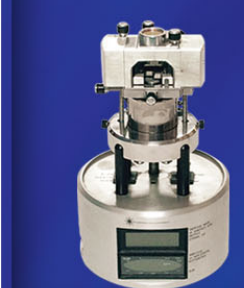

Is your AFM dead

and can't be repaired?

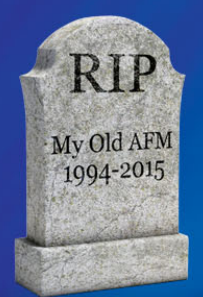

Sick of bad customer support?

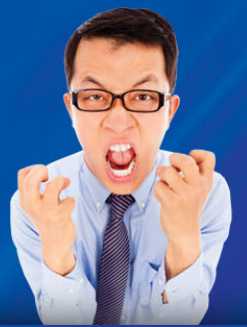

It is time to upgrade your AFM

Minimum $\$ 20,000$ trade-in discount for purchases before August 31st

Asylum Research is today's technology leader in AFM 


\title{
Formation of a hybrid plasmonic waveguide mode probed by dispersion measurement
}

\author{
H. Saito ${ }^{1,2}$ and H. Kurata ${ }^{1, a)}$ \\ ${ }^{1}$ Institute for Chemical Research, Kyoto University, Uji, Kyoto 611-0011, Japan \\ ${ }^{2}$ Quantum Nanoelectronics Research Center, Tokyo Institute of Technology, Oh-Okayama, Meguro-ku, \\ Tokyo 152-8551, Japan
}

(Received 27 January 2015; accepted 23 March 2015; published online 1 April 2015)

\begin{abstract}
Hybrid waveguides, i.e., dielectric waveguides combined with plasmonic waveguides, have great potential for concomitantly exhibiting subwavelength confinement and long range propagation, enabling a highly integrated photonic circuit. We report the characterization of hybrid waveguide modes excited in $\mathrm{Si} / \mathrm{SiO}_{2} / \mathrm{Al}$ films, by dispersion measurement using angle-resolved electron energy-loss spectroscopy. This experiment directly verifies the formation of the hybrid waveguide mode with a strongly localized electromagnetic field in a 6-nm-thick $\mathrm{SiO}_{2}$ layer. The results clearly describe the characteristic behavior of the hybrid waveguide mode, which depends on the effective index of the constituent dielectric waveguide and the surface plasmon-polariton modes. C 2015 AIP Publishing LLC. [http://dx.doi.org/10.1063/1.4916800]
\end{abstract}

\section{INTRODUCTION}

Guiding light with subwavelength confinement is a critical challenge for nanoscale optical applications, such as integrated photonic circuits. ${ }^{1}$ A variety of waveguide structures have been proposed for this purpose in previous reports, ${ }^{2-10}$ some of which have been assessed experimentally. Dielectric waveguide structures like dielectric cylinders can allow light propagation over a long distance, although the confinement is limited by the size of the waveguide structure itself. By contrast, waveguide structures using surface plasmon-polaritons (SPPs), ${ }^{11,12}$ such as a metal surface, can achieve deep subwavelength confinement. However, the propagation loss associated with plasmonic waveguides is high because of electron oscillations in the dissipative metallic regions, ${ }^{12}$ and the propagation distance is usually limited to several microns.

One strategy that has been predicted to be efficient is coupling a dielectric waveguide with a plasmonic waveguide. ${ }^{13-18}$ A hybrid plasmonic waveguide with a dielectric cylinder above a metal surface was proposed, ${ }^{13}$ and its effectiveness as a nanolaser was demonstrated several years ago. ${ }^{19,20}$ The hybrid waveguide structure can store dense energy in the low-permittivity insulator nano-gap between the dielectric waveguide and the metal surface, leading simultaneously to deep subwavelength confinement and long-range propagation. This waveguide mode has been regarded as a coupling of the dielectric waveguide and the SPPs. ${ }^{13,18}$ Although the properties of hybrid waveguide modes have been analyzed theoretically, experimental verifications have so far been lacking. In particular, although the coupled-mode theory suggests that the character of the hybrid waveguide modes depends mainly on the effective indices of the constituent dielectric waveguide and SPP modes, ${ }^{13,18,21}$ this relationship has not yet been confirmed satisfactorily by experiment. In the present work, we show the results of dispersion

\footnotetext{
a) Author to whom correspondence should be addressed. Electronic mail: kurata@eels.kuicr.kyoto-u.ac.jp
}

measurements of the hybrid waveguide mode using angleresolved electron energy-loss spectroscopy (AREELS). ${ }^{22-29}$ In order to investigate the fundamental characteristics of the simplest hybrid waveguide structure, we fabricated threelayered films consisting of a high-permittivity semiconductor layer, a low-permittivity insulator nano-gap (or vacuum) and a metal layer, and a so-called SIM structure. Owing to this simple structure, the dispersion relations observed experimentally can easily be compared with the calculated relationships. Furthermore, the three-layered film with parallel interfaces can be used in real applications, such as integrated photonic circuits, ${ }^{1-17}$ because a structure with a rectangular crosssection is much easier to fabricate using microfabrication technology, e.g., electron beam lithography.

We performed dispersion measurements not only on the SIM films but also on the SI films (without the metal layer) over the energy region encompassing the visible light spectrum to determine the relationship between the effective indices of the constituent modes and the behavior of the hybrid waveguide mode. The low-permittivity insulator gap in the present study was less than $10 \mathrm{~nm}$ thick. The formation of a hybrid waveguide mode with extremely dense electromagnetic energy was confirmed through dispersion measurements.

\section{METHOD}

Figure 1 shows a schematic diagram of AREELS. A narrow slit is placed at the entrance plane of the spectrometer on which the angular dispersive (electron diffraction) pattern is formed. When the slit is set to be perpendicular to the direction of energy dispersion for the spectrometer, the spectral intensity can be obtained as a function of the scattering angle $(\theta)$ and the energy loss $(E)$. Since the scattering angle is proportional to the component of the scattering vector perpendicular to the direction of incident electrons $\left(q_{\perp}\right)$, the observed two-dimensional (2D) AREELS pattern allows us to directly visualize the energy-loss probability as a function of $E$ and $q_{\perp}$ (E-q map), as shown in Fig. 1. When the 


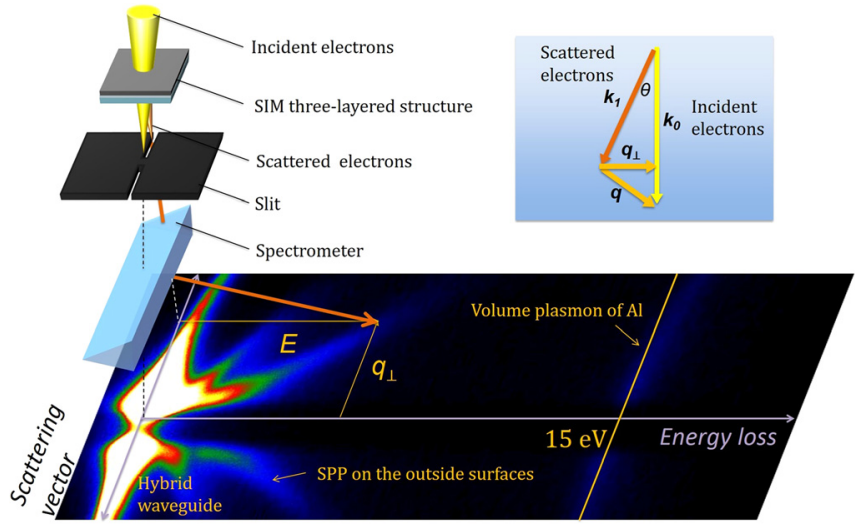

FIG. 1. Schematic diagram of AREELS method. $\mathbf{k}_{\mathbf{0}}$ and $\mathbf{k}_{\mathbf{1}}$ represent the wave vectors of incident and scattered electrons, respectively. An angular selection slit is placed at the entrance plane of the spectrometer to limit electron collection to those scattered perpendicular to the direction of energy dispersion. The gap in the center part of the slit is closed to prevent the intense direct beam from saturating the CCD detector. The inserted E-q map is taken from B2.

incident electrons are normal to the multilayered films, $q_{\perp}$ can be assumed to be the wave vector $k$ (propagation constant) of the propagating waves in waveguide structures. Therefore, the E-q map directly yields the dispersion relations of the waveguide modes.

AREELS measurements were performed at room temperature using a $200 \mathrm{kV}$ transmission electron microscope (JEM-9980 TKP1) equipped with a cold field emission gun and an omega filter as a spectrometer. The energy resolution determined from the full width at half maximum of the zeroloss peak was $0.4 \mathrm{eV}$. In order to achieve the high angular dispersion, the specimen was lifted from the normal position in the image mode with spot illumination. ${ }^{26}$ The scattering vector resolution determined from the full width at half maximum of a direct beam on the E-q map was $5 \times 10^{-3} \mathrm{~nm}^{-1}$. A specimen area $4 \mu \mathrm{m}$ in diameter was illuminated by an incident electron beam with a convergent semi-angle of 6.4 mrad. The E-q maps were measured by a charge-coupled device (CCD) camera with a pixel size corresponding to $27 \mathrm{meV}$ and $3.8 \times 10^{-4} \mathrm{~nm}^{-1}$ along the loss energy and the scattering vector axes in the E-q maps, respectively. Each Eq map was acquired in $10 \mathrm{~s}$. Ten E-q maps taken under the same conditions were superimposed to improve the signalto-noise ratio.

The waveguide structures consisting of the multilayered (SI and SIM) films were prepared as shown in Fig. 2(a). Silicon $(\mathrm{Si})$, silicon dioxide $\left(\mathrm{SiO}_{2}\right)$, and aluminum $(\mathrm{Al})$ were chosen as the semiconductor layer, the insulator gap, and the metal layer, respectively. First, a 6-nm-thick amorphous $\mathrm{SiO}_{2}$ film was produced on the sodium chloride crystal $(\mathrm{NaCl})$ substrate by electron beam evaporation. Then, a non-crystalline $\mathrm{Si}$ film with a thickness of 52,105 , or $157 \mathrm{~nm}$ was deposited on the $\mathrm{SiO}_{2}(6 \mathrm{~nm}) / \mathrm{NaCl}$ substrate by electron beam evaporation. The resulting two-layered films, viz., $\mathrm{Si}(52 \mathrm{~nm}) / \mathrm{SiO}_{2}(6 \mathrm{~nm})$, $\mathrm{Si}(105 \mathrm{~nm}) / \mathrm{SiO}_{2}(6 \mathrm{~nm})$, and $\mathrm{Si}(157 \mathrm{~nm}) / \mathrm{SiO}_{2}(6 \mathrm{~nm})$, are denoted as specimens A1, A2, and A3, respectively. Specimens A1, $\mathrm{A} 2$, and $\mathrm{A} 3$ were floated on the surface of distilled water by dissolving the $\mathrm{NaCl}$ substrates, and transferred onto copper meshes for AREELS measurements. Finally, 35-nm-thick Al films were deposited on the $\mathrm{SiO}_{2}$ side of specimens $\mathrm{A} 1, \mathrm{~A} 2$, and $\mathrm{A} 3$ by thermal evaporation; the resulting specimens will hereafter be denoted as specimens B1, B2, and, B3, respectively. The thickness of each layer was monitored with a quartz oscillator and confirmed by cross-sectional images taken by a scanning transmission electron microscope, as shown in Fig. 2(b). The total thicknesses of the specimens were small enough to be penetrated by the incident electrons. (a)

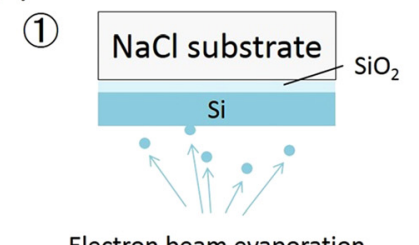

(2)

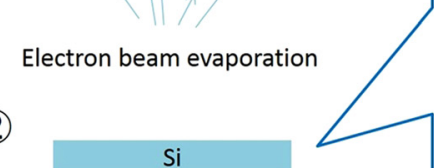

(3)
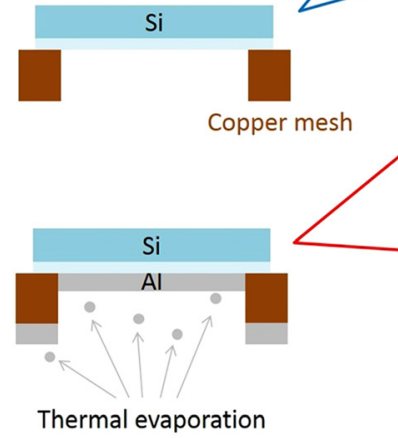

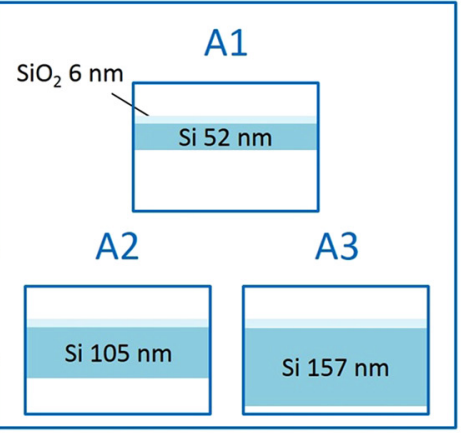

B1

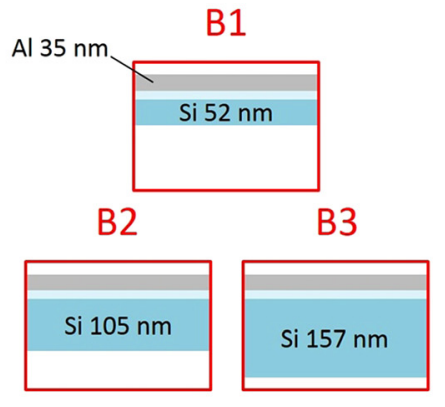

(b)

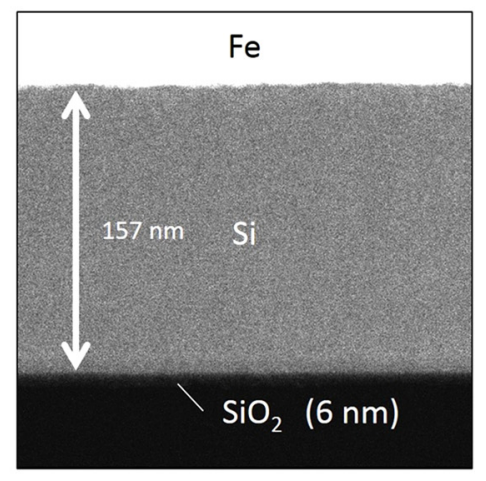

FIG. 2. (a) Schematics of the specimens and the steps of specimen preparation. (b) A cross-sectional STEM image of A3. The brightest region is the iron (Fe) layer deposited to protect the specimen from being damaged by FIB processing. 
Since the SPP mode supported by the plane surface is the transverse magnetic (TM) mode, the hybrid waveguide modes supported by the SIM film are also expected to be only TM modes. Moreover, the incident electrons normal to the plane surface can excite only TM modes. ${ }^{30}$ Therefore, in the present study, we consider the dispersion relations only for TM modes. The experimental E-q maps were compared with the theoretical dispersion curves for TM modes to identify their modes. By solving Maxwell's equations under the appropriate boundary conditions, the dispersion relationship is obtained; it can be expressed in terms of the dielectric function $(\varepsilon)$ and the thickness $(t)$ of each film via the equation

$$
1+K_{m, i} K_{i, s} e^{2 \alpha_{i} t_{i}}+K_{i, s} K_{s, 0} e^{2 \alpha_{s} t_{s}}+K_{m, i} K_{s, 0} e^{2 \alpha_{i} t_{i}} e^{2 \alpha_{s} t_{s}}=0
$$

where

$$
\begin{aligned}
& K_{m, i}=\frac{\frac{\alpha_{m}}{\varepsilon_{m}}+\frac{\alpha_{i}}{\varepsilon_{i}}}{\frac{\alpha_{m}}{\varepsilon_{m}}-\frac{\alpha_{i}}{\varepsilon_{i}}}, \\
& K_{i, s}=\frac{\frac{\alpha_{i}}{\varepsilon_{i}}-\frac{\alpha_{s}}{\varepsilon_{s}}}{\frac{\alpha_{i}}{\varepsilon_{i}}+\frac{\alpha_{s}}{\varepsilon_{s}}}, \\
& K_{s, 0}=\frac{\frac{\alpha_{s}}{\varepsilon_{s}}-\frac{\alpha_{0}}{\varepsilon_{0}}}{\frac{\alpha_{s}}{\varepsilon_{s}}+\frac{\alpha_{0}}{\varepsilon_{0}}} .
\end{aligned}
$$

The indices $\mathrm{m}, \mathrm{i}, \mathrm{s}$, and 0 denote the metal layer (Al), the insulator gap $\left(\mathrm{SiO}_{2}\right)$, the semiconductor layer $(\mathrm{Si})$, and vacuum, respectively. The thickness of the metal layer is assumed to be semi-infinite in this equation. The metal layer changes into vacuum for the dispersion calculation of the SI films. The parameter $\alpha$, i.e., the transverse wave number (damping factor) of the electromagnetic wave perpendicular to the interface, is given by

$$
\alpha_{n}=\sqrt{k^{2}-\varepsilon_{n}\left(\frac{E}{\hbar c}\right)^{2}}(n=m, i, s, 0),
$$

where $\hbar$ and $c$ are the Plank constant divided by $2 \pi$ and the velocity of light in vacuum, respectively.

The dispersion relations were calculated by solving Eq. (1) numerically. The dielectric function of $\mathrm{SiO}_{2}$ determined optically was used for the calculation. ${ }^{31}$ The dielectric function of $\mathrm{Al}$ was assumed to follow the Drude model without damping

$$
\varepsilon_{m}(E)=1-\frac{E_{P}^{2}}{E^{2}},
$$

where $E_{p}$ is the excitation energy for bulk plasmons, which is $15 \mathrm{eV}$ for $\mathrm{Al}^{23}$ The reliability of these dielectric functions of $\mathrm{SiO}_{2}$ and $\mathrm{Al}$ was confirmed in our previous work. ${ }^{28}$ The dielectric function of non-crystalline $\mathrm{Si}$ was determined by the present experiment for the SI films (specimens A2 and $\mathrm{A} 3$ ).

\section{RESULTS}

Figure 3 shows the E-q maps of the SI films (A1, A2, and A3) and SIM films (B1, B2, and B3), in which they are compared with the calculated dispersion relations of light in vacuum, bulk amorphous $\mathrm{SiO}_{2}$, and $\mathrm{Si}$ crystal (white solid lines and curves). Since the real part of the dielectric function of $\mathrm{Si}$ is positive and large in the energy range below $4 \mathrm{eV}$, Čerenkov radiation is generated in Si by $200-\mathrm{keV}$ electrons. This radiation is confined inside the Si slab as discrete waveguide modes ${ }^{27}$ owing to the boundary effect. ${ }^{32}$ Therefore, dispersion curves of $\mathrm{Si}$ waveguide modes would be expected to be observed below $4 \mathrm{eV}$ in the E-q maps of the SI films. In fact, on the E-q map in Fig. 3(b), the dispersion curve of the lowest TM mode $\left(\mathrm{TM}_{0}\right)$ appears in the region between the light lines in vacuum and Si bulk. This is a feature of Si waveguide modes. When the thickness of the Si film is increased, dispersion curves of two TM modes are observed, as shown in Fig. 3(c). The dispersion curve of the $\mathrm{TM}_{0}$ mode shifts to the lower energies with increasing Si thickness. This behavior is characteristic of dielectric waveguide modes. For the thin $\mathrm{Si}$ film (A1), no dispersion curve of the Si waveguide mode was detected below $4 \mathrm{eV}$, as shown in Fig. 3(a). The suppression of the $\mathrm{Si}$ waveguide modes in $\mathrm{A} 1$ can be attributed to the decrease in Čerenkov loss whose probability is roughly proportional to the thickness. ${ }^{33-35}$

On the E-q maps of all SI films shown in Figs. 3(a)-3(c), a broad dispersion curve is observed outside the dispersion relation of light in vacuum above $4 \mathrm{eV}$. This is the dispersion curve for SPPs excited on the $\mathrm{Si} /$ vacuum and $\mathrm{Si} /$ $\mathrm{SiO}_{2}$ interfaces. Since the real part of the dielectric function of $\mathrm{Si}$ has a negative value between $4 \mathrm{eV}$ and the volume plasmon energy (16-17 eV), SPPs can be excited on the interface between $\mathrm{Si}$ and the insulator (or vacuum) in this energy region. The surface on the vacuum side was presumably oxidized slightly because all specimens were exposed to air for a long time before the AREELS experiments. Therefore, the broad dispersion curve above $4 \mathrm{eV}$ is attributed to SPP on two interfaces.

Figures 3(d)-3(f) show the E-q maps measured from SIM films (B1, B2, and B3). The dispersion curves observed below $4 \mathrm{eV}$ are slightly different from those of the SI films. For B1, in particular, a new dispersion curve clearly appears in the E-q map of Fig. 3(d), whereas no dispersion curve was detected for the corresponding SI film (A1), as seen in Fig. 3(a). This dispersion curve might be related to the SPP excited on the $\mathrm{Al} / \mathrm{SiO}_{2}$ interface. Moreover, when the thickness of the Si film in SIM structures is increased, the dispersion curves shift to lower energies (see Figs. 3(e) and 3(f)), similar to the case of SI films. In order to distinguish the dispersion relations of SIM and SI films, their angle-resolved EELS spectra at $q_{\perp}=0.02,0.03$, and $0.04 \mathrm{~nm}^{-1}$ were compared. Figure 4(a) shows the angle-resolved EELS spectra extracted from the E-q maps of Figs. 3(b) and 3(e). The new peaks observed in the SIM film (B2) are lower than those of the $\mathrm{Si}$ waveguide $\left(\mathrm{TM}_{0}\right)$ mode in the SI film (A2), which suggests that the new excitation mode arises from the coupling between the SPPs on the $\mathrm{Al} / \mathrm{SiO}_{2}$ interface and the $\mathrm{TM}_{0}$ modes in the $\mathrm{Si}$ film, representing a so-called hybrid 

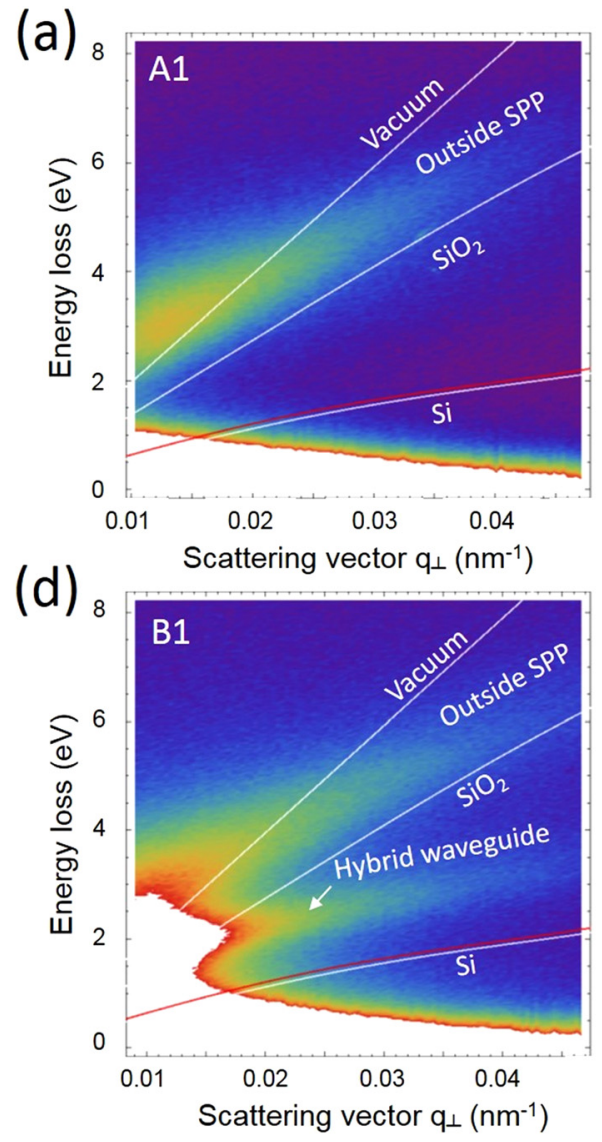

0 (b)

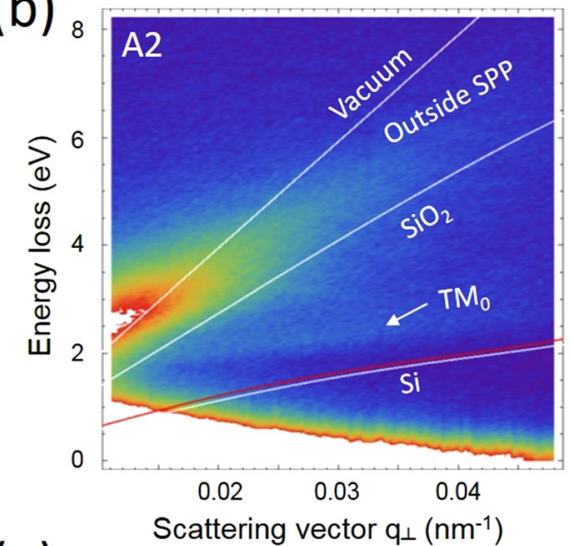

(e) 8

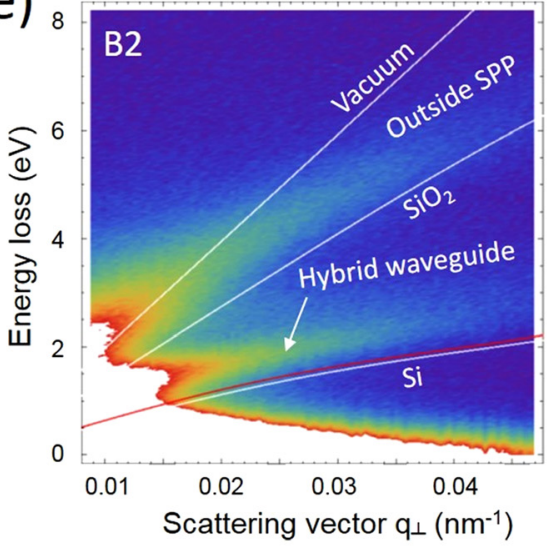

(c)

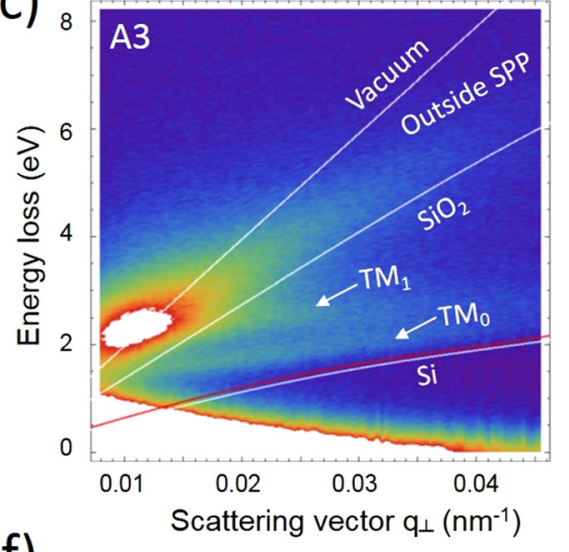

(f)

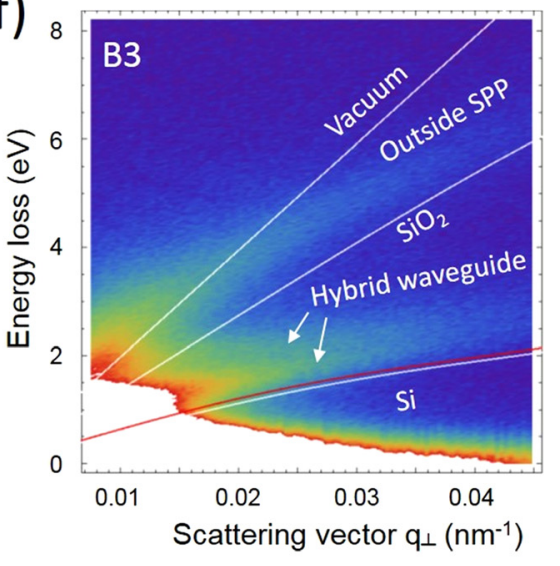

FIG. 3. E-q maps of (a) A1, (b) A2, (c) A3, (d) B1, (e) B2, and (f) B3. The white solid curves and line are the calculated dispersion relations of light in bulk Si, bulk $\mathrm{SiO}_{2}$, and vacuum. The red curve represents the relationship between the energy and $\mathrm{q}_{\perp}$ of Čerenkov radiation excited by $200 \mathrm{keV}$ electrons in bulk $\mathrm{Si}$.

waveguide mode. It should be noted that the energy difference between the hybrid waveguide and the $\mathrm{TM}_{0}$ modes becomes small at large wave vectors $\left(q_{\perp}=0.04 \mathrm{~nm}^{-1}\right)$. Figure 4(b) shows the angle-resolved EELS spectra extracted from the E-q maps of Figs. 3(c) and 3(f), where the second mode of the hybrid waveguide coupled with the $\mathrm{TM}_{1}$ mode of the $\mathrm{Si}$ waveguide also appears as weak shoulder peaks in the spectra of B3. The first and second modes of the hybrid
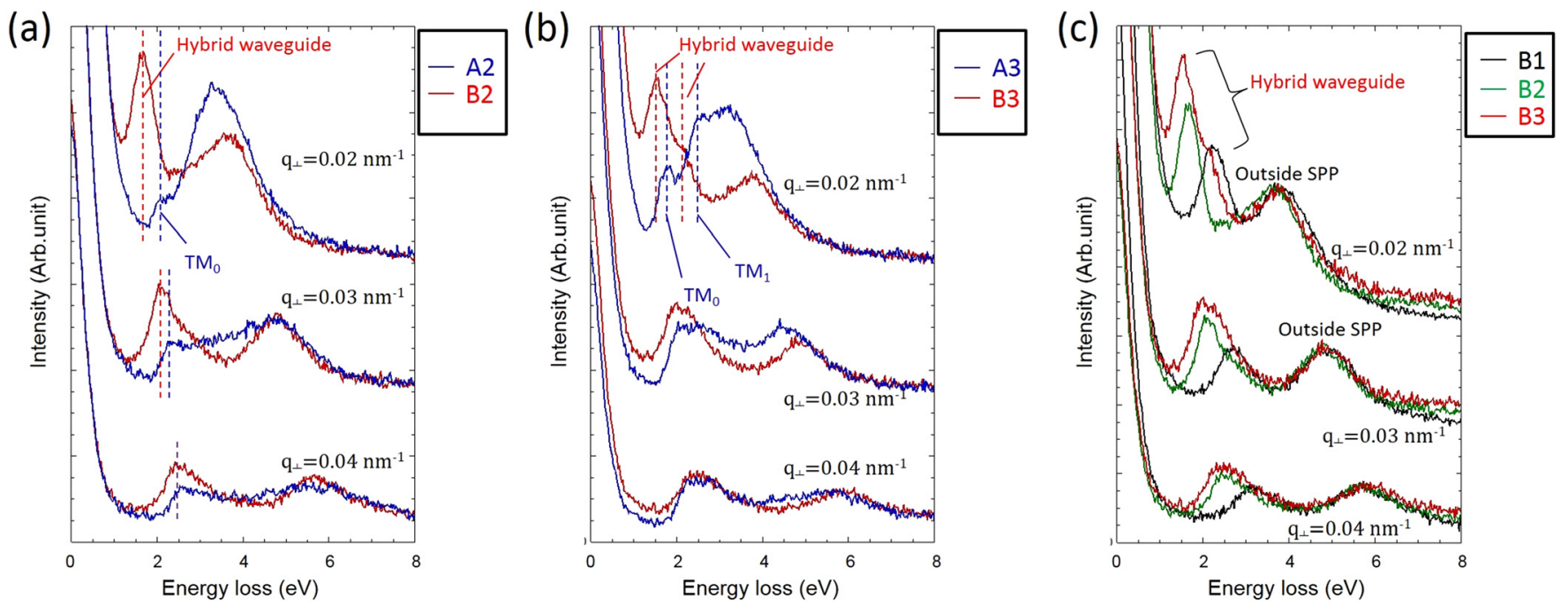

FIG. 4. Angle-resolved EELS obtained from the E-q maps of (a) A2 (blue) and B2 (red), and (b) A3 (blue) and B3 (red) at $q_{\perp}=0.02 \mathrm{~nm}^{-1}, 0.03 \mathrm{~nm}{ }^{-1}$, and $0.04 \mathrm{~nm}^{-1}$. (c) Comparison of the angle-resolved EELS of B1 (black), B2 (green), and B3 (red). 
(a)

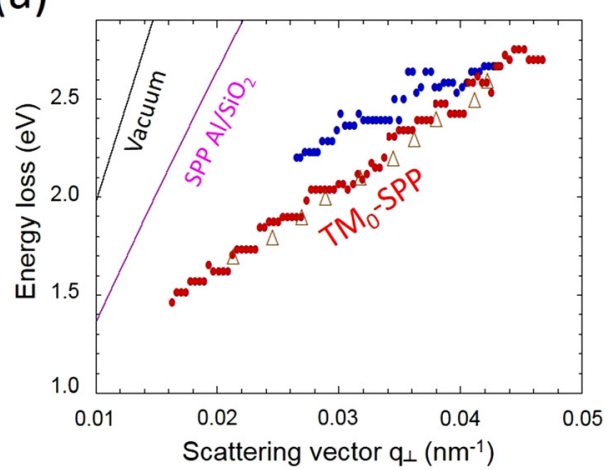

(b)

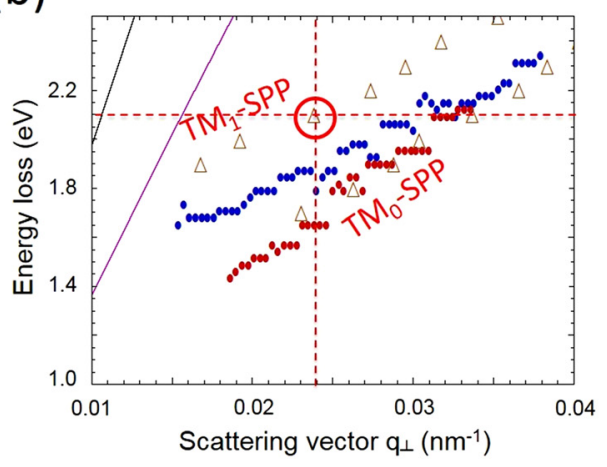

(c)

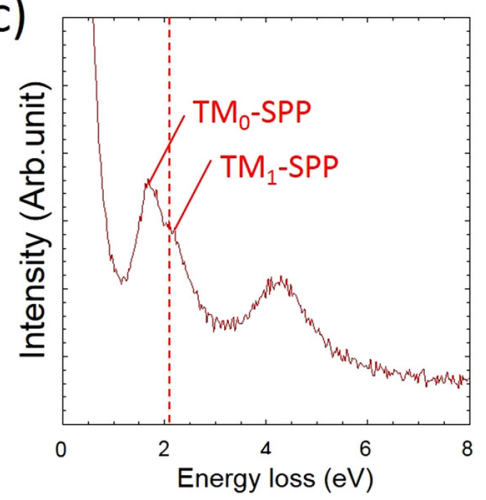

FIG. 5. (a) and (b) Dispersion plots of the hybrid waveguide mode (red filled circles) obtained from (a) B2 and (b) B3, compared with the theoretical dispersion plots (brown open triangles). The dispersion relations of the $\mathrm{TM}_{0}$ mode extracted from $\mathrm{A} 2$ and $\mathrm{A} 3$ are shown by blue filled circles. The solid purple curve and black line are the theoretical dispersion relations of SPPs on the $\mathrm{Al} / \mathrm{SiO}_{2}$ interface and of light in vacuum, respectively. (c) Angle-resolved EELS of $\mathrm{B} 3$ at $q_{\perp}=0.024 \mathrm{~nm}^{-1}$.

waveguide shift to lower energies relative to the $\mathrm{TM}_{0}$ and $\mathrm{TM}_{1}$ modes of the $\mathrm{Si}$ waveguide, respectively. Their differences also decrease with increasing $q_{\perp}$.

The angle-resolved EELS spectra extracted from the E-q maps of all SIM structures are compared in Fig. 4(c). The intensity of each spectrum is normalized at the peak of the outside SPPs. It should be noted that the ratio of excitation probability of the hybrid waveguide modes to that of the outside SPPs clearly increases with increasing Si layer thickness. This behavior is typical of volume excitations like Cerenkov radiation. ${ }^{33-35}$ Thus, the intensity increases with $\mathrm{Si}$ thickness is attributed to the enhancement of the hybrid waveguide modes by coupling with Čerenkov radiation, similar to the abovementioned case for $\mathrm{Si}$ waveguide modes.

For accurate assignment of the hybrid waveguide modes, the dispersion relation observed experimentally should be compared to that calculated by solving Eq. (1) numerically. Figures 5(a) and 5(b) compare experimental and calculated dispersion relations. The experimental dispersions (red filled circles) were extracted from the E-q maps in Figs. 3(e) and $3(\mathrm{f})$ by finding a local maximum in the energy region from $1.45 \mathrm{eV}$ to $2.75 \mathrm{eV}$ and $2.15 \mathrm{eV}$, respectively. These experimental plots agree well with the calculated dispersion (red open triangles) of the hybrid waveguide mode formed from the coupling of the $\mathrm{TM}_{0}$ waveguide and SPP mode $\left(\mathrm{TM}_{0}\right.$-SPP mode) with a quasi-even symmetry. For comparison, these figures also plot the dispersion relations of the $\mathrm{Si}$ waveguide $\mathrm{TM}_{0}$ mode extracted from Figs. 3(b) and 3(c) (see blue filled circles). It can be seen that the dispersion for the $\mathrm{TM}_{0}-\mathrm{SPP}$ mode merges with that of the $\mathrm{TM}_{0}$ mode at large wave vectors. Also seen in Fig. 5(b) on the high-energy side is the theoretical dispersion for the $\mathrm{TM}_{1}-\mathrm{SPP}$ mode with quasi-even symmetry. Although it is difficult to extract the experimental dispersion relation for the $\mathrm{TM}_{1}$-SPP mode from the E-q map of Fig. 3(f), the angle-resolved spectrum for $q_{\perp}=0.024 \mathrm{~nm}^{-1}$ has a shoulder at $2.1 \mathrm{eV}$ as shown in Fig. 5(c). The position of this shoulder agrees with the theoretical prediction shown in Fig. 5(b), so the existence of the $\mathrm{TM}_{1}$-SPP mode with quasi-even symmetry is verified experimentally.

\section{DISCUSSION}

Hybrid waveguide modes have been demonstrated in SIM waveguide structures in the visible wavelength region by measuring the dispersion relations directly using AREELS. The dispersion curve of the $\mathrm{TM}_{0}$-SPP hybrid mode shown in Fig. 5(a) is distinct from those of the $\mathrm{TM}_{0}$ and SPP modes at low energy, but merged with that of the $\mathrm{TM}_{0}$ mode at high energy. This suggests that the character of a hybrid waveguide mode depends on the energy. A hybrid mode can be characterized in terms of the effective index, defined as the propagation constant of waveguide mode divided by the wave vector in vacuum. Since the normal component of the scattering vector is equal to the propagation constant, it is easy to convert an E-q map into a dispersion relation of the effective index.

Figure 6(a) shows the effective indices for the $\mathrm{TM}_{0}$-SPP hybrid mode, the $\mathrm{TM}_{0}$ mode supported by a $\mathrm{Si}$ film, and the SPP mode supported by an aluminum film as a function of energy, which is converted from the E-q relationship of Fig. 5 (a). Since the effective index for the $\mathrm{TM}_{0}$ mode below $2.2 \mathrm{eV}$ could not be determined experimentally owing to the weak intensity in the E-q map of Fig. 4(b), it was calculated by using the dielectric function evaluated from the results of Fig. 3(c). The effective indices for the $\mathrm{TM}_{0}$ and SPP modes intersect at about $1.8 \mathrm{eV}$, which is the critical energy where the effective index of the $\mathrm{TM}_{0}$-SPP hybrid mode differs greatly from the average of the effective indices of the $\mathrm{TM}_{0}$ and SPP modes. The SPPs at the $\mathrm{Al} / \mathrm{SiO}_{2}$ interface and the polarized charges in the $\mathrm{Si}$ waveguide oscillate in phase at this critical energy.

A simple coupling mode theory suggests that the maximum coupling occurs at this critical energy and the character of the hybrid mode is governed equally by both modes, ${ }^{13,21}$ so that it can be expected that the electromagnetic energy will be confined at the insulator gap region effectively. The electromagnetic energy density per unit area in the propagation plane is given by the following equation: ${ }^{13}$

$$
W=\frac{1}{2}\left(\frac{d(\varepsilon \omega)}{d \omega}|\mathbf{E}|^{2}+\mu_{0}|\mathbf{H}|^{2}\right),
$$


(a)

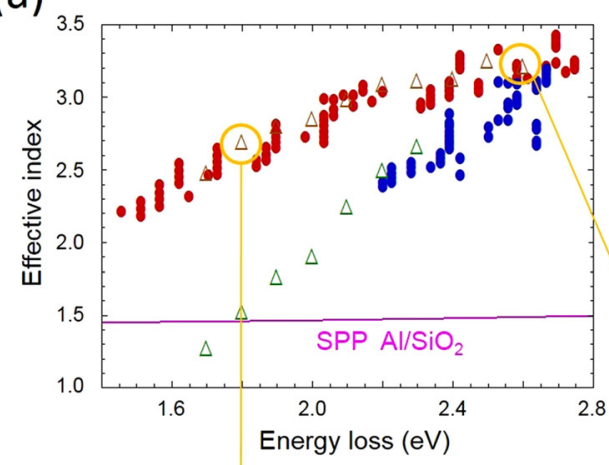

(c)

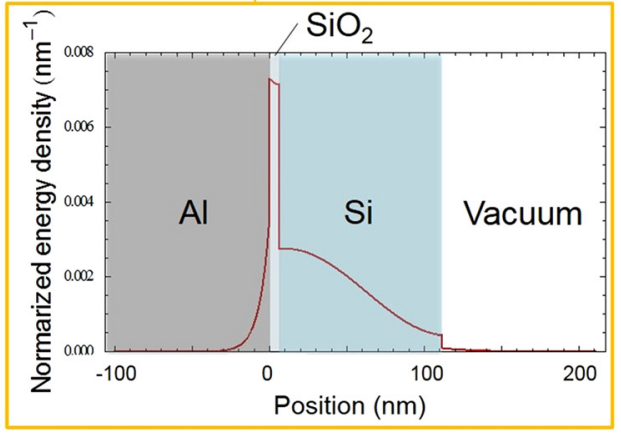

(b)

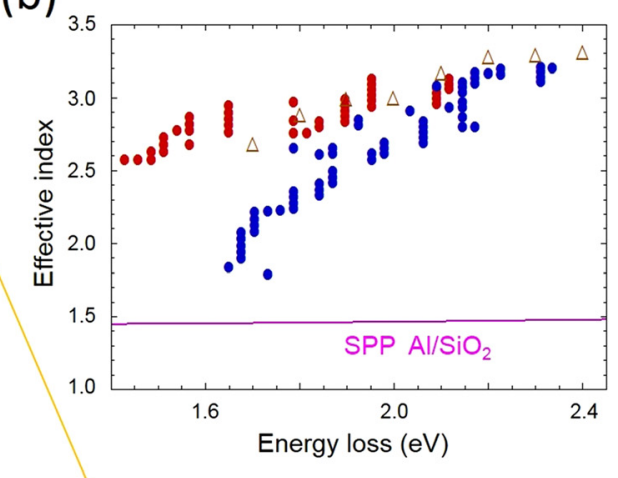

(d)

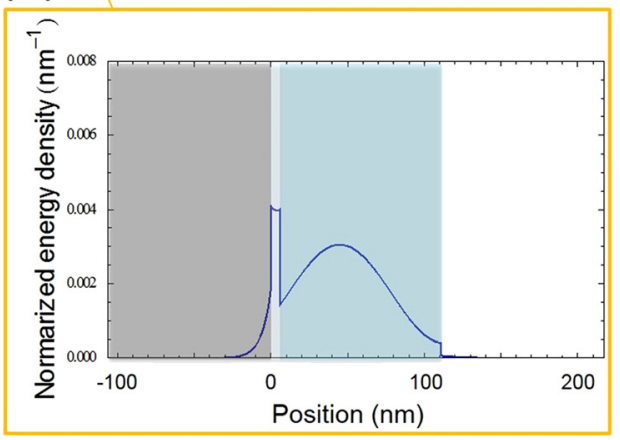

FIG. 6. (a) and (b) Energy dependence of the effective indices for (a) A2 and B2, and (b) A3 and B3 calculated from the dispersion plots (Figs. 5(a) and $5(b))$. The red and blue filled circles represent the $\mathrm{TM}_{0}$-SPP hybrid mode and the $\mathrm{TM}_{0}$ mode obtained in the AREELS experiment, respectively. The brown and green open triangles represent the theoretical indices. The solid purple curve is the theoretical indices of SPP on the $\mathrm{Al} / \mathrm{SiO}_{2}$ interface. (c) and (d) Normalized electromagnetic energy density for the $\mathrm{TM}_{0^{-}}$ SPP mode in B2 calculated from the theoretical dispersion relation (c) near the intersection $(1.8 \mathrm{eV})$ and $(\mathrm{d})$ on the high energy side $(2.6 \mathrm{eV})$. where $\omega$ and $\mu_{0}$ are the angular frequency and permeability of vacuum, respectively. The expressions for the electric field $\mathbf{E}$ and magnetic field $\mathbf{H}$ are given by solutions of Eq. (1). Figure 6(c) shows the electromagnetic energy density calculated for the SIM structure (specimen B2) at $1.8 \mathrm{eV}$, which is normalized by the total electromagnetic energy calculated by the integration of $W$ along the normal to the interface. The characteristics of the hybrid waveguide mode with a strong energy confinement at the gap region can be seen in this figure. With increasing energy, the difference between the effective indices for the $\mathrm{TM}_{0}$-SPP hybrid mode and the $\mathrm{TM}_{0}$ mode becomes small, which means that the hybrid mode becomes $\mathrm{TM}_{0}$-like. Indeed, the electromagnetic energy density calculated at $2.6 \mathrm{eV}$ (Fig. 6(d)) shows a larger contribution in the Si waveguide region and a smaller energy confinement at the gap region, as compared to Fig. 6(c). At energies below $1.8 \mathrm{eV}$, the effective index for the $\mathrm{TM}_{0}$ mode is smaller than that for the SPP mode, and the hybrid mode is expected to become SPP-like. The dispersion for the effective index for the SIM structure of specimen B3 with a thick $\mathrm{Si}$ layer is shown in Fig. 6(b). With increasing $\mathrm{Si}$ waveguide layer thickness, the critical energy giving maximum coupling seems to be decrease, which suggests that the energy of effective hybrid coupling can be controlled by adjusting the thickness of the dielectric waveguide layer.

\section{CONCLUSION}

The present dispersion measurements conducted on $\mathrm{Si} /$ $\mathrm{SiO}_{2} / \mathrm{Al}$ structures by AREELS demonstrated that the SPP mode excited in the interface of $\mathrm{Al} / \mathrm{SiO}_{2}$ can couple with the $\mathrm{TM}_{0}$ waveguide mode excited in the Si layer, leading to the $\mathrm{TM}_{0}$-SPP hybrid waveguide mode. The dispersion of the effective indices for the constituent modes showed that the maximum coupling occurs at the critical energy. Our experiment with SIM structures having different semiconductor thicknesses indicated that the critical energy for a hybrid waveguide mode decreased with increasing thickness. The electromagnetic energy of a hybrid waveguide mode excited at the critical energy was found to be confined effectively to the insulator gap region. Our results clearly demonstrated that the character and critical energy of a hybrid waveguide mode can be controlled by adjusting the thickness of the semiconductor layer. Finally, we verified the $\mathrm{TM}_{1}$-SPP mode with quasi-even symmetry experimentally.

${ }^{1}$ D. K. Gramotnev and S. I. Bozhevolnyi, Nat. Photonics 4, 83 (2010).

${ }^{2}$ J. Takahara, S. Yamagishi, H. Taki, A. Morimoto, and T. Kobayashi, Opt. Lett. 22, 475 (1997).

${ }^{3}$ P. Berini, Phys. Rev. B 63, 125417 (2001).

${ }^{4}$ K. Tanaka and M. Tanaka, Appl. Phys. Lett. 82, 1158 (2003).

${ }^{5}$ A. D. Boardman, G. C. Aers, and R. Teshima, Phys. Rev. B 24, 5703 (1981).

${ }^{6}$ I. V. Novikov and A. A. Maradudin, Phys. Rev. B 66, 035403 (2002).

${ }^{7}$ V. R. Almeida, Q. Xu, C. A. Barrios, and M. Lipson, Opt. Lett. 29, 1209 (2004).

${ }^{8}$ R. Zia, J. A. Schuller, and M. L. Brongersma, Phys. Rev. B 74, 165415 (2006).

${ }^{9}$ D. F. P. Pile, T. Ogawa, D. K. Gramotnev, T. Okamoto, M. Haraguchi, M. Fukui, and S. Matsuo, Appl. Phys. Lett. 87, 061106 (2005).

${ }^{10}$ S. I. Bozhevolnyi, V. S. Volkov, E. Devaux, and T. W. Ebbesen, Phys. Rev. Lett. 95, 046802 (2005).

${ }^{11}$ R. H. Ritchie, Phys. Rev. 106, 874 (1957).

${ }^{12}$ W. L. Barnes, A. Dereux, and T. W. Ebbesen, Nature 424, 824 (2003).

${ }^{13}$ R. F. Oulton, V. J. Sorger, D. A. Genov, D. F. P. Pile, and X. A. Zhang, Nat. Photonics 2, 496 (2008).

${ }^{14}$ R. Salvador and A. Martínez, IEEE J. Sel. Top. Quantum Electron. 14, 1496 (2008).

${ }^{15}$ M. Fujii, J. Leuthold, and W. Freude, IEEE Photonics Technol. Lett. 21, 362 (2009).

${ }^{16}$ D. Dai and S. He, Opt. Express 17, 16646 (2009).

${ }^{17}$ M. Z. Alam, J. Meier, J. S. Aitchison, and M. Mojahedi, Opt. Express 18, 12971 (2010). 
${ }^{18}$ M. Z. Alam, J. S. Aitchison, and M. Mojahedi, IEEE J. Sel. Top. Quantum Electron. 19, 4602008 (2013)

${ }^{19}$ R. F. Oulton, V. J. Sorger, T. Zentgraf, R. M. Ma, C. Gladden, L. Dai, G. Bartal, and X. Zhang, Nature 461, 629 (2009).

${ }^{20}$ R. M. Ma, R. F. Oulton, V. J. Sorger, G. Bartal, and X. Zhang, Nat. Mater. 10, 110 (2011).

${ }^{21}$ A. W. Snyder and J. D. Love, Optical Waveguide Theory (Chapman and Hall, London, New York, 1983).

${ }^{22}$ H. Watanabe, J. Phys. Soc. Jpn. 11, 112 (1956).

${ }^{23}$ R. B. Pettit, J. Silcox, and R. Vincent, Phys. Rev. B 11, 3116 (1975).

${ }^{24}$ C. H. Chen, J. Silcox, and R. Vincent, Phys. Rev. B 12, 64 (1975).

${ }^{25}$ P. A. Midgley, Ultramicroscopy 76, 91 (1999).

${ }^{26}$ M. K. Kinyanjui, C. Kramberger, T. Pichler, J. C. Meyer, P. Wachsmuth, G. Benner, and U. Kaiser, Europhys. Lett. 97, 57005 (2012).
${ }^{27}$ H. Saito, C. H. Chen, and H. Kurata, J. Appl. Phys. 113, 113509 (2013).

${ }^{28}$ H. Saito, K. Namura, M. Suzuki, and H. Kurata, Microscopy 63, 85 (2014).

${ }^{29}$ S. C. Liou, C.-S. Shie, C. H. Chen, R. Breitwieser, W. W. Pai, G. Y. Guo, and M.-W. Chu, Phys. Rev. B 91, 045418 (2015).

${ }^{30}$ E. Kröger, Z. Phys. 235, 403 (1970).

${ }^{31}$ H. R. Philipp, J. Phys. Chem. Solids 32, 1935 (1971).

${ }^{32}$ F. J. Garcia de Abajo, A. Rivacoba, N. Zabala, and N. Yamamoto, Phys. Rev. B 69, 155420 (2004).

${ }^{33}$ M. Stöger-Pollach, H. Franco, P. Schattschneider, S. Lazar, B. Schaffer, W. Grogger, and H. W. Zandbergen, Micron 37, 396 (2006).

${ }^{34}$ L. Gu, V. Srot, W. Sigle, C. Koch, P. van Aken, F. Scholz, S. B. Thapa, C. Kirchner, M. Jetter, and M. Rühle, Phys. Rev. B 75, 195214 (2007).

${ }^{35}$ R. Erni and N. D. Browning, Ultramicroscopy 108, 84 (2008). 